\title{
Predictive value of the surgical Apgar score on postoperative complications in advanced gastric cancer patients treated with neoadjuvant chemotherapy followed by radical gastrectomy: a single-center retrospective study
}

Masato Hayashi, Takaki Yoshikawa*, Masahiro Yura, Sho Otsuki, Yukinori Yamagata, Shinji Morita, Hitoshi Katai and Toshirou Nishida

\begin{abstract}
Background: The surgical Apgar score (SAS) or modified SAS (mSAS) has been reported as a simple and easy risk assessment system for predicting postoperative complications in primary surgery for gastric cancer. However, few studies have described the SAS's utility in gastric surgery after neoadjuvant chemotherapy (NAC).

Methods: One hundred and fifteen patients who received NAC and radical gastrectomy from 2008 and 2015 were included in this study. The SAS was determined by the estimated blood loss (EBL), lowest intraoperative mean arterial pressure, and lowest heart rate. The mSAS was determined by the EBL reassessed using the interquartile values. The predictive values of the SAS/mSAS for postoperative complications were assessed with univariate and multiple logistic regression analyses.
\end{abstract}

Results: Among the 115 patients, 41 (35.7\%) developed postoperative complications. According to analyses with receiver operating characteristic curves of the SAS and mSAS for predicting postoperative complications, the cut-off value of the mSAS was set at 8 . The rates of anastomotic leakage, pancreatic fistula, and arrhythmia in patients with high mSAS $(\geq 8)$ values were higher than in those with low (0-3) and moderate [1-4] mSAS values. A multiple logistic regression analysis showed that the operation time, body mass index, and diabetes mellitus were independent risk factors for postoperative complications. The mSAS was not a significant predictor.

Conclusion: The predictive value of SAS or mSAS for morbidity may be limited in patients who undergo gastric cancer surgery after NAC. Future prospective studies with a large sample size will be needed to confirm the present results.

Keywords: Gastrectomy, Neoadjuvant chemotherapy, Surgical Apgar score, Gastric cancer, Postoperative complication

\footnotetext{
* Correspondence: tayoshik@ncc.go.jp

Department of Gastric Surgery, National Cancer Center Hospital, 5-1-1 Tsukiji,

Chuo-ku, Tokyo 104-0045, Japan
}

(c) The Author(s). 2020 Open Access This article is licensed under a Creative Commons Attribution 4.0 International License, which permits use, sharing, adaptation, distribution and reproduction in any medium or format, as long as you give appropriate credit to the original author(s) and the source, provide a link to the Creative Commons licence, and indicate if changes were made. The images or other third party material in this article are included in the article's Creative Commons licence, unless indicated otherwise in a credit line to the material. If material is not included in the article's Creative Commons licence and your intended use is not permitted by statutory regulation or exceeds the permitted use, you will need to obtain permission directly from the copyright holder. To view a copy of this licence, visit http://creativecommons.org/licenses/by/4.0/. The Creative Commons Public Domain Dedication waiver (http://creativecommons.org/publicdomain/zero/1.0/) applies to the data made available in this article, unless otherwise stated in a credit line to the data. 


\section{Background}

Gastrectomy with lymphadenectomy is a core treatment strategy for curing gastric cancer [5]. Although several chemotherapy regimens, such as adjuvant chemotherapy or palliative chemotherapy, have been shown to be effective [6,7], the prognosis of advanced gastric cancer is still unsatisfactory [1]. In such situations, neoadjuvant chemotherapy (NAC) may be another attractive treatment for improving the prognosis of patients with advanced disease [2-4]; however, surgery after NAC is associated with technical difficulties due to fibrosis induced by chemotherapy, which may cause morbidity. Because surgical complications can not only induce mortality but also decrease a patient's quality of life, the prediction of morbidity is quite important.

Previously, the surgical Apgar score (SAS) was reported as an easy and simple predictor of postoperative complications in various operations, including gastrectomy [8-11]. SAS is determined by the following intraoperative factors: the estimated blood loss (EBL), lowest intraoperative mean arterial pressure (LMAP), and lowest heart rate (LHR). Furthermore, the modified SAS (mSAS) using a cut-off value of EBL has previously been proposed for assessing the postoperative complication risk in several types of surgery as well [12-15]. However, previous studies investigating the SAS or mSAS only examined gastric cancer patients who received primary surgery with or without adjuvant chemotherapy. Therefore, whether or not the SAS or mSAS are sensitive predictors for gastric cancer patients undergoing surgery after NAC remains unclear.

This study investigated the utility of the SAS and mSAS for predicting morbidity of advanced gastric cancer patients treated with NAC and gastrectomy.

\section{Methods}

\section{Participants}

The medical records of patients at National Cancer Center Hospital, Tokyo, Japan, were retrospectively reviewed to select advanced gastric cancer patients who were treated with NAC and radical gastrectomy from January 2008 to December 2015. The inclusion criteria were [5] gastric adenocarcinoma without previous treatment, [6] a histologically proven diagnosis of adenocarcinoma, and [7] patients who received NAC and radical gastrectomy as a treatment. R1 (Residual Tumor 1) or R2 resection (Residual Tumor 2) cases were excluded from this study. $\mathrm{R} 1 / \mathrm{R} 2$ were defined as microscopic/macroscopic residual tumor (positive resection margin or cytology positive), respectively according to the Japanese Gastric Cancer Association Classification [16].

Although NAC has not been established as a standard treatment strategy yet in Japan, the regimens of NAC were basically determined as follows: (1) large type 3 or type 4 gastric cancer was treated according to the protocol of JCOG0501; (2) cT3/cT4 with any $\mathrm{N}$ was treated according to the protocol of the in-house study of S-1 and Oxaliplatin; and (3) para-aortic lymph node swelling or extensive nodal swelling along the major branched arteries (bulky-N) was treated according to the protocol of JCOG0405 or JCOG1002.

Following the protocol of JCOG 0405 and JCOG1002, gastrectomy with D2 plus para-aortic nodal dissection was performed. Other trials needed D2 lymphadenectomy. The histological tumor regression of primary tumor was evaluated with the Japanese Classification of Gastric Carcinoma (JCGC) [17].

\section{The definitions of SAS and mSAS}

The SAS was calculated based on the three intraoperative factors of EBL, LMAP, and LHR (Table 1) [8]. The score was calculated from the total points of each category. We also calculated the mSAS in accordance with the method of Miki et al. [12]. In the mSAS, the EBL cut-off value was determined based on its quartile values among the included patients. The median EBL was 519 $\mathrm{ml}$, the 25 th percentile was $305 \mathrm{ml}$, and the 75 th percentile was $960 \mathrm{ml}$ (Table 2).

\section{The complication severity evaluation}

Postoperative complications were defined as any morbidity occurring within 30 days after surgery. The severity was graded using the Clavien-Dindo Classification $[18,19]$. In this study, only complications of grade $\geq$ IIIa were considered postoperative complications.

\section{Statistical analyses}

The SPSS software program (IBM, Armonk, NY, USA; 25.0 version) was used for all analyses of this study. A nonparametric test (Mann-Whitney $\mathrm{U}$ test) was

Table 1 Surgical Apgar Score (SAS)

\begin{tabular}{|c|c|c|c|c|c|}
\hline & 0 point & 1 point & 2 points & 3 points & 4 points \\
\hline Estimated blood loss (mL) & $>1000$ & $601-1000$ & $101-600$ & $\leq 100$ & - \\
\hline Lowest mean arterial pressure $(\mathrm{mmHg})$ & $<40$ & $40-54$ & $55-69$ & $\geq 70$ & - \\
\hline Lowest heart rate (beats/min) & $>85$ & $76-85$ & $66-75$ & $56-65$ & $\leq 55$ \\
\hline
\end{tabular}

Surgical Apgar score was calculated with sum of the points for each category in the course of the procedure 
Table 2 Modified Surgical Apgar Score (mSAS)

\begin{tabular}{|c|c|c|c|c|c|}
\hline & 0 point & 1 point & 2 points & 3 points & 4 points \\
\hline Estimated blood loss (mL) & $>960$ & $519-960$ & $305-518$ & $\leq 304$ & - \\
\hline Lowest mean arterial pressure $(\mathrm{mmHg})$ & $<40$ & $40-54$ & $55-69$ & $\geq 70$ & - \\
\hline Lowest heart rate (beats/min) & $>85$ & $76-85$ & $66-75$ & $56-65$ & $\leq 55$ \\
\hline
\end{tabular}

modified Surgical Apgar Score was calculated with sum of the points as well

performed for continuous values, whilst Pearson's chisquared test or Fisher's exact test was used for categorical values. A multiple logistic regression analysis was performed to determine the correlation between postoperative complications and perioperative factors in patients who were treated with NAC and radical gastrectomy. Analyses with a receiver operating characteristic (ROC) curve were performed to evaluate the utility of the SAS and mSAS for predicting postoperative complications and to determine the optimum cut-off values of the scores. $P$ values of $<$ 0.05 were recognized statistically significant in 2tailed statistical tests.

\section{Results}

\section{Postoperative complications}

One hundred and twenty patients were treated with NAC followed by radical gastrectomy. Four patients were excluded due to R1 resection, and one patient was excluded due to adenosquamous carcinoma. This study thus ultimately included 115 patients. Among them, 41 patients (35.7\%) developed surgical morbidity. As for the incidence of postoperative complications, it was 47 (40.9\%). The details of postoperative complications are shown in Table 3.
Determination of the cut-off values for predicting postoperative complications

We created the ROC curves of the SAS and mSAS for predicting postoperative complications (data not shown). Because the area under the curve (AUC) of the mSAS was higher than that of the SAS, a further analysis was done using the mSAS. The cut-off value for mSAS was set at 8 based on the curve for predicting complications in this population (AUC of SAS: 0.589, AUC of mSAS: $0.631)$.

\section{Clinicopathological features of this population}

Table 4 shows the clinicopathological features of this population between low mSAS $(<8)$ and high mSAS $(\geq$ 8 ). The body mass index (BMI), operation time, and EBL were significantly greater in the low mSAS group than in the high mSAS group.

Table 5 shows the risk stratification of complications according to the mSAS, which was divided into 3 categories: low mSAS, 0-3; moderate mSAS, 4-7; high mSAS, 8-10. The risk of complication in the high mSAS group was significantly lower than that in the low mSAS group. Table 6 shows that the rates of pancreatic fistula, anastomotic leakage, and arrhythmia were significantly higher in the low mSAS group than in the moderate and high mSAS groups.

Table 3 Details of Postoperative Complications

\begin{tabular}{|c|c|c|c|c|c|c|}
\hline \multirow[t]{2}{*}{ Complications } & \multicolumn{5}{|c|}{ Grade according to Clavien Dindo Classification } & \multirow[t]{2}{*}{ Total (\%) } \\
\hline & IIla & Illb & $\mathrm{IVa}$ & $\mathrm{IVb}$ & V & \\
\hline Pancreatic fistula & 21 & 0 & 0 & 0 & 0 & $21(18.3 \%)$ \\
\hline Anastomotic leakage & 2 & 2 & 0 & 0 & 0 & $4(3.5 \%)$ \\
\hline Abdominal abscess & 3 & 0 & 0 & 0 & 0 & $3(2.6 \%)$ \\
\hline Duodenal stump fistula & 1 & 1 & 0 & 0 & 1 & $3(2.6 \%)$ \\
\hline Pneumonia & 1 & 0 & 0 & 0 & 0 & $1(0.9 \%)$ \\
\hline Bleeding & 3 & 1 & 0 & 0 & 0 & $4(3.5 \%)$ \\
\hline Lymph fistula & 1 & 0 & 0 & 0 & 0 & $1(0.9 \%)$ \\
\hline Acute kidney failure & 0 & 0 & 1 & 0 & 0 & $1(0.9 \%)$ \\
\hline Arrhythmia & 0 & 0 & 1 & 0 & 0 & $1(0.9 \%)$ \\
\hline Others & 7 & 1 & 0 & 0 & 0 & $8(7.0 \%)$ \\
\hline Total & 39 & 5 & 2 & 0 & 1 & 47 (40.9\%) \\
\hline
\end{tabular}


Table 4 Patients and clinicopathological features between low and high mSAS

\begin{tabular}{|c|c|c|c|c|}
\hline Variables & All patients $N=115$ & $\begin{array}{l}\text { low mSAS }<8 \\
83(72.2 \%)\end{array}$ & $\begin{array}{l}\text { high } m S A S \geq 8 \\
32(27.8 \%)\end{array}$ & $p$ value \\
\hline Age (yr) & $63.3( \pm 10.8)$ & $63.2( \pm 10.8)$ & $63.8( \pm 10.9)$ & 0.736 \\
\hline Gender & & & & 0.044 \\
\hline Male & $81(70.4 \%)$ & $63(75.9 \%)$ & $18(56.3 \%)$ & \\
\hline Female & $34(29.6 \%)$ & $20(24.1 \%)$ & $14(43.8 \%)$ & \\
\hline Body Mass Index (BMI) & $22.3( \pm 3.3)$ & $22.7( \pm 3.6)$ & $21.1( \pm 2.8)$ & 0.027 \\
\hline $\mathrm{BMI} \geq 22$ & $60(52.2 \%)$ & $46(55.4 \%)$ & $14(43.8 \%)$ & 0.001 \\
\hline Diabetes Mellitus & $9(7.8 \%)$ & 7 (8.4\%) & $2(6.3 \%)$ & 1 \\
\hline Smoking history & $73(63.5 \%)$ & $58(69.9 \%)$ & 15 (46.9\%) & 0.030 \\
\hline $\begin{array}{l}\text { American Society of Anesthesiologists } \\
\text { Physical Status (ASA-PS) }\end{array}$ & & & & 0.703 \\
\hline 1 & $11(9.6 \%)$ & $7(8.4 \%)$ & $4(12.5 \%)$ & \\
\hline 2 & $94(81.7 \%)$ & $68(81.9 \%)$ & $26(81.3 \%)$ & \\
\hline 3 & $10(8.7 \%)$ & $8(9.6)$ & $2(6.3 \%)$ & \\
\hline Tumor location ${ }^{a}$ & & & & 0.097 \\
\hline Upper & $46(40 \%)$ & $38(45.8 \%)$ & $8(25.0 \%)$ & \\
\hline Middle & $39(33.9 \%)$ & $26(31.3 \%)$ & 13 (40.6\%) & \\
\hline Lower & $20(17.4 \%)$ & $11(13.3 \%)$ & 9 (28.1\%) & \\
\hline Whole & $10(8.7 \%)$ & $8(9.6 \%)$ & $2(6.3 \%)$ & \\
\hline ycStage & & & & 0.302 \\
\hline । & $3(2.6 \%)$ & $3(3.6 \%)$ & 0 & \\
\hline$\|$ & 47 (40.9\%) & $31(37.3 \%)$ & $16(50.0 \%)$ & \\
\hline III & $65(56.5 \%)$ & $49(59.0 \%)$ & $16(50.0 \%)$ & \\
\hline Neoadjuvant chemotherapy regimen & & & & 0.057 \\
\hline $\mathrm{S}-1^{\mathrm{b}}$ and cisplatin & $74(64.3 \%)$ & $51(61.4 \%)$ & $23(71.9 \%)$ & \\
\hline $\mathrm{S}-1^{\mathrm{b}}$ and oxaliplatin & $14(12.2 \%)$ & $8(9.6 \%)$ & $6(18.8 \%)$ & \\
\hline $\mathrm{S}-1^{\mathrm{b}}$, docetaxel and cisplatin & $13(11.3 \%)$ & $10(12.0 \%)$ & $3(9.4 \%)$ & \\
\hline Others & $14(12.2 \%)$ & $14(16.9 \%)$ & 0 & \\
\hline Surgical Procedure & & & & 0.001 \\
\hline Distal gastrectomy & $29(25.2 \%)$ & $13(15.7 \%)$ & $16(50.0 \%)$ & \\
\hline Total gastrectomy & $83(72.3 \%)$ & $68(81.9 \%)$ & $15(46.9 \%)$ & \\
\hline Other & $3(2.6 \%)$ & $2(2.4 \%)$ & $1(3.1 \%)$ & \\
\hline Operation time (min) & $337.1( \pm 111.1)$ & $365.6( \pm 113.5)$ & $263.4( \pm 59.8)$ & $<0.001$ \\
\hline Surgical Apgar score & $6.25( \pm 1.1)$ & $5.77( \pm 1.2)$ & $7.50( \pm 0.67)$ & $<0.001$ \\
\hline modified Surgical Apgar Score & $6.34( \pm 1.5)$ & $5.65( \pm 1.2)$ & $8.16( \pm 0.46)$ & $<0.001$ \\
\hline Estimated blood loss (ml) & $711.5( \pm 627.0)$ & $898.1( \pm 644.3)$ & $227.6( \pm 112.9)$ & $<0.001$ \\
\hline Lowest mean arterial pressure & $55.6( \pm 6.6)$ & $54.8( \pm 6.5)$ & $57.6( \pm 6.4)$ & 0.034 \\
\hline Lowest heart rate & $58.7( \pm 8.4)$ & $59.8( \pm 8.8)$ & $56.0( \pm 6.7)$ & 0.034 \\
\hline Extent of lymphadenectomy & & & & 0.023 \\
\hline $\mathrm{D} 2^{\mathrm{c}}$ & $48(41.7 \%)$ & $31(37.3 \%)$ & $17(53.1 \%)$ & \\
\hline$D 2+{ }^{d}$ & $40(34.8 \%)$ & $27(32.5 \%)$ & $13(40.6 \%)$ & \\
\hline$D 3^{e}$ & $27(23.5 \%)$ & $25(30.1 \%)$ & $2(6.3 \%)$ & \\
\hline $\mathrm{ypT}^{\mathrm{a}}$ factor & & & & 0.346 \\
\hline 0 & $4(3.5 \%)$ & $3(3.6 \%)$ & $1(3.1 \%)$ & \\
\hline T1a & $1(0.9 \%)$ & $1(1.2 \%)$ & 0 & \\
\hline
\end{tabular}


Table 4 Patients and clinicopathological features between low and high mSAS (Continued)

\begin{tabular}{|c|c|c|c|c|}
\hline Variables & All patients $N=115$ & $\begin{array}{l}\text { low mSAS }<8 \\
83(72.2 \%)\end{array}$ & $\begin{array}{l}\text { high } m S A S \geq 8 \\
32(27.8 \%)\end{array}$ & $p$ value \\
\hline $\mathrm{T1b}$ & $13(11.3 \%)$ & $10(12.0 \%)$ & $3(9.4 \%)$ & \\
\hline $\mathrm{T} 2$ & $17(14.8 \%)$ & $15(18.1 \%)$ & $10(31.3 \%)$ & \\
\hline T3 & $51(44.3 \%)$ & $36(43.4 \%)$ & 15 (46.9\%) & \\
\hline T4a & $25(21.7 \%)$ & $15(18.1 \%)$ & $10(31.3 \%)$ & \\
\hline T4b & $4(3.5 \%)$ & $3(3.6 \%)$ & $1(3.1 \%)$ & \\
\hline $\mathrm{ypN}^{\mathrm{a}}$ factor & & & & 0.781 \\
\hline 0 & 39 (33.9\%) & $30(36.1 \%)$ & 9 (28.1\%) & \\
\hline 1 & $24(20.9 \%)$ & $18(21.7 \%)$ & $6(18.8 \%)$ & \\
\hline 2 & $26(22.6 \%)$ & 19 (22.9\%) & 7 (21.9\%) & \\
\hline 3 & $26(22.6 \%)$ & $16(19.3 \%)$ & $10(31.3 \%)$ & \\
\hline M factor & & & & 0.725 \\
\hline 0 & $104(90.4 \%)$ & $74(89.2 \%)$ & $30(93.8 \%)$ & \\
\hline 1 & $11(9.6 \%)$ & $9(10.8 \%)$ & $2(6.3 \%)$ & \\
\hline ypStage $e^{a}$ & & & & 0.392 \\
\hline 0 & $3(2.6 \%)$ & $3(3.6 \%)$ & 0 & \\
\hline । & 19 (16.5\%) & $16(19.3 \%)$ & $3(9.4 \%)$ & \\
\hline$\|$ & 37 (32.3\%) & $25(30.1 \%)$ & $12(37.5 \%)$ & \\
\hline III & 45 (39.1\%) & $30(36.1 \%)$ & $15(46.9 \%)$ & \\
\hline IV & $11(9.6 \%)$ & $9(10.8 \%)$ & $2(6.3 \%)$ & \\
\hline postoperative complication ${ }^{f} \geq I \| l a$ & $41(35.7 \%)$ & $35(42.2 \%)$ & $6(18.8 \%)$ & 0.029 \\
\hline Histological response of primary lesion & & & & 0.673 \\
\hline 0 & $2(1.7 \%)$ & $2(2.4 \%)$ & 0 & \\
\hline $1 a$ & $33(28.7 \%)$ & $24(28.9 \%)$ & $9(28.1 \%)$ & \\
\hline $1 b$ & $30(26.1 \%)$ & $22(26.5 \%)$ & $8(25.0 \%)$ & \\
\hline 2 & $47(40.9 \%)$ & $32(38.6 \%)$ & $15(46.9 \%)$ & \\
\hline 3 & $3(2.6 \%)$ & $3(3.6 \%)$ & 0 & \\
\hline
\end{tabular}

${ }^{a}$ According to the seventh edition of the International Union Against Cancer tumor, node, metastasis (TNM) classification system

${ }^{\mathrm{b}}$ Tegafur/Gimeracil/Oteracil: Product name is TS-1

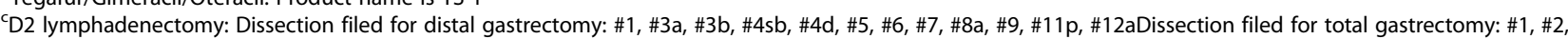

\#3a, \#3b, \#4sa, \#4sb, \#4d, \#5, \#6, \#7, \#8a, \#9, \#10, \#11p, \#11d, \#12a

${ }^{\mathrm{d} D} 2+$ lymphadenectomy: D2 plus either \#16a2/\#16b1

'D3 lymphadenectomy: D2 plus both \#16a2 and \#16b1

${ }^{\mathrm{f}}$ According to Clavien-Dindo Classification

\section{Results of a logistic regression analysis of the factors associated with postoperative complications}

Table 7 shows the results of univariate and multiple logistic regression analyses for postoperative complications. The univariate analysis revealed significant differences in the gender, BMI, diabetes mellitus (DM), smoking history, ycStage, operation time, EBL and mSAS. Except for EBL, which was excluded because it was an apparent confounding factor for the mSAS, these factors were included as covariates in a multiple logistic regression analysis of factors predicting complications. In the multiple logistic regression analysis, the BMI, presence of DM, and operation time were detected as independent risk factors for postoperative complications. The mSAS was not identified as a risk factor.

\section{Results of an analysis of confounding factors for the mSAS}

Confounding factors of surgical outcome and mSAS were considered. An analysis of the confounding factors revealed that the low mSAS group included significantly greater proportions of patients with a high BMI and long operation time than the high mSAS group (Table 4). 
Table 5 Risk stratification of complication according to mSAS

\begin{tabular}{lllll}
\hline & with complications & OR $(95 \% \mathrm{Cl})$ & \\
& $N=41(35.7 \%)$ & without complications & \\
& & & & \\
mSAS 0-3 & $3(7.3 \%)$ & $1(1.4 \%)$ & 1 & \\
mSAS 4-7 & $32(78.0 \%)$ & $47(63.5 \%)$ & $0.23(0.023-2.280)$ & 0.208 \\
mSAS 8-10 & $6(14.6 \%)$ & $26(35.1 \%)$ & $0.08(0.007-0.875)$ & $\mathbf{0 . 0 3 9}$ \\
\hline
\end{tabular}

${ }^{a}$ Grade Illa or higher According to Clavien-Dindo Classification

\section{Discussion}

Before obtaining the results of our study, SAS and mSAS have been considered predictors for surgical morbidity in patients who receive surgery for gastric cancer. However, whether or not this remains true in patients who receive neoadjuvant chemotherapy followed by surgery is unclear. Unexpectedly, this study failed to demonstrate the utility of the SAS or mSAS for predicting postoperative complications in this patient population. The mSAS was not an independent predictor of morbidity. To our knowledge, this study is the first report to evaluate the correlation between the SAS and complications in patients treated with NAC followed by radical gastrectomy.

Several previous studies have demonstrated the utility of SAS for predicting complications [9, 10], although other studies have described the usefulness of the mSAS but not the SAS $[12,14]$. The utility of the SAS seems to depend on the type of surgery or the characteristics of the cohort. We considered the reasons why the SAS and mSAS were not found to be risk factors for complications in the present study. In our study, a high BMI, the presence of $\mathrm{DM}$, and a long operation time were identified as independent risk factors for postoperative complications. In the low mSAS group, the proportions of patients with a high BMI and long operation time were higher than in the high mSAS group, suggesting that these factors were confounders of the mSAS. These results suggested that patients with a high BMI or longer operation time easily develop surgical morbidities and that the predictive value of the mSAS was inferior to that of a high BMI or long operation time.

Anesthesia may also have influenced the results. The LHR and LMAP, which are included in the SAS and mSAS, can easily be affected by anesthesia. Deep

Table 6 Comparison of complication ${ }^{\mathrm{a}}$ among categorized mSAS

\begin{tabular}{lllll}
\hline & mSAS 0-3 & $\begin{array}{l}\text { mSAS 4-7 } \\
N=79\end{array}$ & $\begin{array}{l}\text { mSAS 8-10 } \\
N=32\end{array}$ & $p$ value \\
\hline Pancreatic fistula & $2(50.0 \%)$ & $17(21.5 \%)$ & $2(6.3 \%)$ & 0.042 \\
anastomotic leakage & $1(25.0 \%)$ & $3(3.8 \%)$ & $0(0 \%)$ & 0.035 \\
arrhythmia & $1(25.0 \%)$ & $0(0 \%)$ & $0(0 \%)$ & $<0.001$ \\
\hline
\end{tabular}

${ }^{a}$ Grade Illa or higher According to Clavien-Dindo Classification anesthesia can reduce the arterial pressure without any bleeding, and the use of high doses of opioids can prevent an increase in the heart rate caused by bleeding or dehydration. Ideally, the mSAS should be evaluated under the same conditions of anesthesia. Unfortunately, however, anesthesia in our institution was managed by several doctors under different policies during the study period. The lack of statistical significance of the SAS and mSAS for predicting complications might therefore have been influenced by these different management approaches.

This study and Miki's [12] study differ in some respects, although both studies investigated the predictive value of the mSAS in gastric cancer patients. First, the EBL of our population was much higher than that in Miki's study, possibly due to surgical difficulties, such as fibrosis induced by NAC or extensive lymphadenectomy, which was selected in more than half of the present cohort. These differences might have caused our results to differ from those of Miki's study. Second, our cut-off value of mSAS was much higher than that in the previous report, even though our EBL was higher, possibly due to the very high incidence of complications in the present population. This point might be another reason for the differences between the present and previous study.

In this study, a high BMI, the presence of DM, and a long operation time were detected as risk predictors for postoperative complications in patients treated with NAC. These factors are well known predictors of complications in primary surgery [20-23]. A high BMI is closely related to excessive visceral fat, which may extend the operation time and impair lymph node dissection [24, 25]. Thus, a high BMI increases the difficulty of the whole operation. Excessive visceral fat easily induces metabolic syndrome, including DM, which makes patients more susceptible to infection and can inhibit wound healing. A long operation time has been reported to be a risk factor of postoperative complications, accelerating the speed of body metabolism and increasing the consumption of nutrition [22]. These factors would not be changed after NAC.

Several limitations associated with the present study warrant mention. First, this was a retrospective, singlecenter study. Although our study population was mostly limited to patients enrolled in prospective clinical trials, 
Table 7 Univariate analysis and multiple logistic regression analysis for postoperative complications

\begin{tabular}{|c|c|c|c|c|c|c|}
\hline \multirow[b]{2}{*}{ Variables } & \multicolumn{3}{|l|}{ Univariate analysis } & \multicolumn{3}{|c|}{ Multiple logistic regression analysis } \\
\hline & $\begin{array}{l}\text { with complications }{ }^{\text {b }} \\
N=41(35.7 \%)\end{array}$ & $\begin{array}{l}\text { without complications }{ }^{\mathrm{b}} \\
N=74(64.3 \%)\end{array}$ & $\overline{p \text { value }}$ & $\overline{\mathrm{OR}}$ & $95 \% \mathrm{Cl}$ & $p$ value \\
\hline Gender & & & 0.034 & 0.85 & $0.24-3.02$ & 0.82 \\
\hline Male & $34(82.9 \%)$ & $47(63.5 \%)$ & & & & \\
\hline Female & $7(17.1 \%)$ & $27(36.5 \%)$ & & & & \\
\hline Body Mass Index (BMI) & $24.4( \pm 3.2)$ & $21.1( \pm 2.7)$ & $<0.001$ & 3.74 & $1.33-10.51$ & 0.012 \\
\hline Diabetes Mellitus & $7(17.1 \%)$ & $2(2.7 \%)$ & 0.010 & 13.5 & $2.00-91.0$ & 0.008 \\
\hline Smoking history & $32(78.0 \%)$ & $41(55.4 \%)$ & 0.017 & 2.39 & $0.74-7.70$ & 0.145 \\
\hline ycStage ${ }^{a}$ & & & 0.020 & 1.69 & $0.62-4.57$ & 0.303 \\
\hline । & $0(0 \%)$ & $3(4.1 \%)$ & & & & \\
\hline$\|$ & $11(26.8 \%)$ & $36(48.6 \%)$ & & & & \\
\hline$\|$ & $30(73.2 \%)$ & $35(47.3 \%)$ & & & & \\
\hline Operation time (min) & $395.7( \pm 138.3)$ & $304.7( \pm 76.2)$ & $<0.001$ & 4.07 & $1.36-12.12$ & 0.012 \\
\hline mSAS & $5.88( \pm 1.6)$ & $6.61( \pm 1.4)$ & 0.018 & 0.9 & $0.63-1.27$ & 0.54 \\
\hline Estimated blood loss (ml) & $899.7( \pm 788.1)$ & $607.2( \pm 492.7)$ & 0.011 & - & - & - \\
\hline Lowest mean arterial pressure & $56.2( \pm 6.4)$ & $55.3( \pm 6.7)$ & 0.057 & - & - & - \\
\hline Lowest heart rate & $60.7( \pm 8.2)$ & $57.6( \pm 8.4)$ & 0.492 & - & - & - \\
\hline
\end{tabular}

${ }^{a}$ According to the seventh edition of the International Union Against Cancer tumor, node, metastasis (TNM) classification system

${ }^{\mathrm{b}} \mathrm{Grade}$ Illa or higher According to Clavien-Dindo Classification

the possibility of several biases could not be completely excluded. Furthermore, we cannot deny the possibility of type 2 error in this study because of the small sample size. For these reasons, a prospective study with a large sample size is needed to confirm our results. Second, the sample size was small, so the predictive value of the mSAS might have been underestimated in this study.

\section{Conclusion}

The predictive value of SAS or mSAS for morbidity may be limited in patients who undergo gastric cancer surgery after NAC. A prospective study with a large sample size will be needed to confirm the present results.

\section{Abbreviations \\ SAS: Surgical Apgar score; mSAS: modified SAS; NAC: Neoadjuvant chemotherapy; EBL: Estimated blood loss; LMAP: Lowest mean arterial pressure; LHR: Lowest heart rate; ROC: Receiver operating characteristics; JCGC: Japanese Classification of Gastric Carcinoma; DM: Diabetes mellitus; BMI: Body mass index; ASA-PS: American Society of Anesthesiologists Performance Status}

\section{Acknowledgements}

We are deeply grateful to Ms. Yumi Yoshida, who is a member of the staff at National Cancer Center Hospital, Department of Gastric Surgery. Without her help, this paper would not have materialized.

\section{Authors' contributions}

$\mathrm{MH}$ and TY designed the study. And $\mathrm{MH}$ is the first author of this manuscript. TY also checked the manuscript and advised the first author on the preparation of the manuscript. MY contributed to collection of data and approved the version to be submitted and any revised versions. SO contributed to collection of data and approved the version to be submitted and any revised versions. YY contributed to collection of data and approved the version to be submitted and any revised versions. SM contributed to collection of data and approved the version to be submitted and any revised versions. HK contributed to collection of data and approved the version to be submitted and any revised versions. TN contributed to collection of data and approved the version to be submitted and any revised versions. The author(s) read and approved the final manuscript.

Funding

This present study does not contain funding.

\section{Availability of data and materials}

The datasets used and analyzed during the current study are available from the corresponding author on reasonable request.

\section{Ethics approval and consent to participate}

This present study conforms to the Declaration of Helsinki and was approved by the Institutional Review Board (IRB) of the National Cancer Center Hospital (No: 2017-077).

This is a retrospective study and no consent to participate to study was required.

\section{Consent for publication}

Not applicable.

\section{Competing interests}

There are no competing interests.

Received: 2 April 2020 Accepted: 5 July 2020

Published online: 11 July 2020

References

1. Digklia A, Wagner AD. Advanced gastric cancer: current treatment landscape and future perspectives. World J Gastroenterol. 2016;22(8):2403-14.

2. Tsuburaya A, Nagata N, Cho H, Hirabayashi N, Kobayashi M, Kojima H, et al. Phase II trial of paclitaxel and cisplatin as neoadjuvant chemotherapy for locally advanced gastric cancer. Cancer Chemother Pharmacol. 2013;71(5): 1309-14.

3. Yoshikawa T, Sasako M, Yamamoto S, Sano T, Imamura H, Fujitani K, et al. Phase II study of neoadjuvant chemotherapy and extended surgery for locally advanced gastric cancer. Br J Surg. 2009;96(9):1015-22. 
4. Tsuburaya A, Mizusawa J, Tanaka Y, Fukushima N, Nashimoto A, Sasako M, et al. Neoadjuvant chemotherapy with S-1 and cisplatin followed by D2 gastrectomy with Para-aortic lymph node dissection for gastric cancer with extensive lymph node metastasis. Br J Surg. 2014;101(6):653-60.

5. Sasako M. Principles of surgical treatment for curable gastric cancer. J Clin Oncol. 2003;21(23Suppl):2.

6. Bang Y-J, Kim Y-W, Yang H-K, Chung HC, Park Y-K, Lee KH, et al. Adjuvant capecitabine and oxaliplatin for gastric cancer after D2 gastrectomy (CLAS SIC): a phase 3 open-label, randomised controlled trial. Lancet. 2012; 379(9813):315-21.

7. Kang YK, Kang WK, Shin DB, Chen J, Xiong J, Wang J, et al. Capecitabine/ cisplatin versus 5 -fluorouracil/cisplatin as first-line therapy in patients with advanced gastric cancer: a randomised phase III noninferiority trial. Ann Oncol. 2009;20(4):666-73.

8. Gawande AA, Kwaan MR, Regenbogen SE, Lipsitz SA, Zinner MJ. An Apgar score for surgery. J Am Coll Surg. 2007;204(2):201-8.

9. Yamada T, Tsuburaya A, Hayashi T, Aoyama T, Fujikawa H, Shirai J, et al. Surgical Apgar score predicts postoperative complications after surgery for gastric cancer. Glob Surg. 2015;1(3):48-51.

10. La Torre M, Ramacciato G, Nigri G, Balducci G, Cavallini M, Rossi M, et al. Post-operative morbidity and mortality in pancreatic surgery. The role of surgical Apgar score. Pancreatology. 2013;13(2):175-9.

11. Nair A, Bharuka A, Rayani BK. The Reliability of Surgical Apgar Score in Predicting Immediate and Late Postoperative Morbidity and Mortality: A Narrative Review. Rambam Maimonides Med J. 2018;9(1):e0004.

12. Miki Y, Tokunaga M, Tanizawa Y, Bando E, Kawamura T, Terashima M. Perioperative risk assessment for gastrectomy by surgical apgar score. Ann Surg Oncol. 2014;21(8):2601-7.

13. Day KE, Prince AC, Lin CP, Greene BJ, Carroll WR. Utility of the modified surgical Apgar score in a head and neck Cancer population. Otolaryngol Head Neck Surg. 2018;159(1):68-75.

14. Xing XZ, Wang HJ, Qu SN, Huang CL, Zhang H, Wang $H$, et al. The value of esophagectomy surgical apgar score (eSAS) in predicting the risk of major morbidity after open esophagectomy. J Thorac Dis. 2016;8(7):1780-7.

15. Pearson ACS, Subramanian A, Schroeder DR, Findlay JY. Adapting the surgical Apgar score for perioperative outcome prediction in liver transplantation: a retrospective study. Transplant Direct. 2017:3(11):e221.

16. Association JGC. Japanese classification of gastric carcinoma: 3rd English edition. Gastric Cancer. 2011:14:101-12.

17. Sano T, Aiko T. Japanese gastric cancer treatment guidelines 2010 (ver. 3). Gastric Cancer. 2011;14(2):97-100

18. Clavien PA, Barkun J, de Oliveira ML, Vauthey JN, Dindo D, Schulick RD, et al. The Clavien-Dindo classification of surgical complications: five-year experience. Ann Surg. 2009;250(2):187-96.

19. Katayama H, Kurokawa $Y$, Nakamura K, Ito H, Kanemitsu Y, Masuda N, et al. Extended Clavien-Dindo classification of surgical complications: Japan clinical oncology group postoperative complications criteria. Surg Today. 2016;46(6):668-85.

20. Wu WW, Zhang WH, Zhang WY, Yang L, Deng XQ, Zhu T. Risk factors of the postoperative 30-day readmission of gastric cancer surgery after discharge: a PRISMA-compliant systematic review and meta-analysis. Medicine (Baltimore). 2019:98(10):e14639.

21. Martin AN, Das D, Turrentine FE, Bauer TW, Adams RB, Zaydfudim VM. Morbidity and mortality after Gastrectomy: identification of modifiable risk factors. J Gastrointest Surg. 2016;20(9):1554-64

22. Wang X, Yao Y, Qian H, Li H, Zhu X. Longer operating time during Gastrectomy has adverse effects on short-term surgical outcomes. J Surg Res. 2019;243:151-9.

23. Jínek TAL, Vrba R, Duda M, Škrovina M. Risk factors and post-operative complications after gastrectomy for cancer. Rozhl Chir. 2018;97(8):10.

24. Eom BW, Joo J, Yoon HM, Ryu KW, Kim YW, Lee JH. A body shape index has a good correlation with postoperative complications in gastric cancer surgery. Ann Surg Oncol. 2014;21(4):1115-22.

25. Ri M, Aikou S, Seto Y. Obesity as a surgical risk factor. Ann Gastroenterol Surg. 2018;2(1):13-21.

\section{Publisher's Note}

Springer Nature remains neutral with regard to jurisdictional claims in published maps and institutional affiliations.

\section{Ready to submit your research? Choose BMC and benefit from:}

- fast, convenient online submission

- thorough peer review by experienced researchers in your field

- rapid publication on acceptance

- support for research data, including large and complex data types

- gold Open Access which fosters wider collaboration and increased citations

- maximum visibility for your research: over $100 \mathrm{M}$ website views per year

At BMC, research is always in progress.

Learn more biomedcentral.com/submissions 